\title{
Polarization Selective Magnetic Vortex Dynamics and Core Reversal in Rotating Magnetic Fields
}

\author{
Michael Curcic, ${ }^{1, *}$ Bartel Van Waeyenberge,${ }^{1,2,+}$ Arne Vansteenkiste, ${ }^{2}$ Markus Weigand, ${ }^{1}$ Vitalij Sackmann, ${ }^{1}$ \\ Hermann Stoll, ${ }^{1, *}$ Manfred Fähnle, ${ }^{1}$ Tolek Tyliszczak, ${ }^{3}$ Georg Woltersdorf, ${ }^{4}$ Christian H. Back, ${ }^{4}$ and Gisela Schütz ${ }^{1}$ \\ ${ }^{1}$ Max-Planck-Institut für Metallforschung, 70569 Stuttgart, Germany \\ ${ }^{2}$ Department of Subatomic and Radiation Physics, Ghent University, 9000 Gent, Belgium \\ ${ }^{3}$ Advanced Light Source, LBNL, 94720 Berkeley, California, USA \\ ${ }^{4}$ Institut für Experimentelle und Angewandte Physik, Universität Regensburg, 93040 Regensburg, Germany \\ (Received 1 June 2008; revised manuscript received 1 September 2008; published 3 November 2008)
}

\begin{abstract}
We report on the observation of magnetic vortex dynamics in response to rotating magnetic fields in submicron platelets. Unlike linear fields or spin polarized currents, which excite both vortex core polarization states, an in-plane rotating field can selectively excite one of the polarization states. We demonstrate by direct imaging with time-resolved scanning x-ray microscopy that the rotating field only excites the gyrotropic mode if the rotation sense of the field coincides with the vortex gyration sense and that such a field can selectively reverse the vortex polarization.
\end{abstract}

DOI: 10.1103/PhysRevLett.101.197204

PACS numbers: 75.40.Gb, 75.60.Jk, 75.75.+a

A detailed understanding of the static and dynamic properties of ferromagnetic thin film structures is crucial for their successful implementation in technological devices, like magnetic sensors or magnetic memories. Nanoscaled magnetic thin film structures like magnetic wires or platelets are also interesting objects to study the fundamental dynamics of magnetic configurations like domain walls and vortices.

In thin film structures of soft magnetic materials, the stray field is minimized by forcing the magnetization in the sample plane and forming inhomogeneous configurations like, e.g., domain walls. In micron and submicron sized structures of suitable thickness, this can result in a stable magnetic vortex configuration. The in-plane curling magnetization in such elements turns out of the plane at the center, avoiding a singularity and forming the vortex core. The vortex core has only a radius of about 5-15 nanometers [1,2], but plays a key role in the magnetization dynamics. The out-of-plane magnetization of the vortex core (called "polarization," $p$ ) can point in two directions, "up" or "down" $(p=+1$ or $p=-1$, respectively). $p$ defines together with the sense of the in-plane flux closure (called "circulation," $c ; c=+1$ for counterclockwise magnetization, $c=-1$ for clockwise magnetization), the magnetic configuration. The combination of circulation and polarization also gives the vortex a handedness (right-handed for $c p=+1$, left-handed for $c p=-1$ ).

According to the equation of motion for magnetic structures, introduced by Thiele [3], and applied to vortex structures by Huber [4], a moving vortex experiences an in-plane gyro force perpendicular to its velocity and of which the sign is given by the core polarization. This gives rise to a gyration counterclockwise for $p=+1$ and clockwise for $p=-1$. When a vortex is displaced from its equilibrium position, the restoring force from the demagnetizing fields and the gyro force results in a spiral motion back into the equilibrium position. This motion corresponds to the fundamental excitation mode of the vortex structure, with a frequency typically in the sub-GHz range, and can be directly excited by an alternating magnetic field. This mode was first observed more than 20 years ago [5], but the recent advances in magnetic microscopy have revealed unexpected details. It was discovered that the excitation of this mode can toggle the vortex core polarization [6].

In-plane uniaxial excitations, like linear oscillating fields, do not break the symmetry of mirror-symmetric structures such as squares or disks, and would result in symmetric dynamics for vortices of opposite polarizations. The resulting switching process should be symmetric, too: the excitation simply toggles the polarization state back and forth and thus the final state should depend on both the amplitude and the duration of excitation [6]. This is different for in-plane rotating fields, which couple differently to the two polarization states [7] and hence lead to an asymmetric switching behavior [8-10].

In this Letter, we report on the experimental observation of the vortex dynamics due to in-plane rotating magnetic fields. $500 \times 500 \times 50 \mathrm{~nm}^{3}$ square Permalloy (Py, $\mathrm{Ni}_{80} \mathrm{Fe}_{20}$ ) elements with a magnetic vortex configuration were excited by an in-plane rotating magnetic field at a frequency close to the gyrotropic eigenfrequency and imaged by time-resolved magnetic x-ray microscopy (see Fig. 1).

The elements were patterned on top of two crossing copper striplines, $150 \mathrm{~nm}$ thick and $12 \mu \mathrm{m}$ wide (cf. Fig. 1) using $e$-beam lithography and a lift-off process. To ensure transparency for soft $\mathrm{x}$ rays, the striplines and Py elements were prepared on a $100 \mathrm{~nm}$ thick $\mathrm{Si}_{3} \mathrm{~N}_{4}$ membrane. An in-plane rotating magnetic field $\mathbf{B}(t)=$ $\left(B_{0} \cos (\omega t), B_{0} \sin (\omega t), 0\right)$ with frequency $|\omega /(2 \pi)|$ and amplitude $B_{0}$ was generated by running two oscillating 

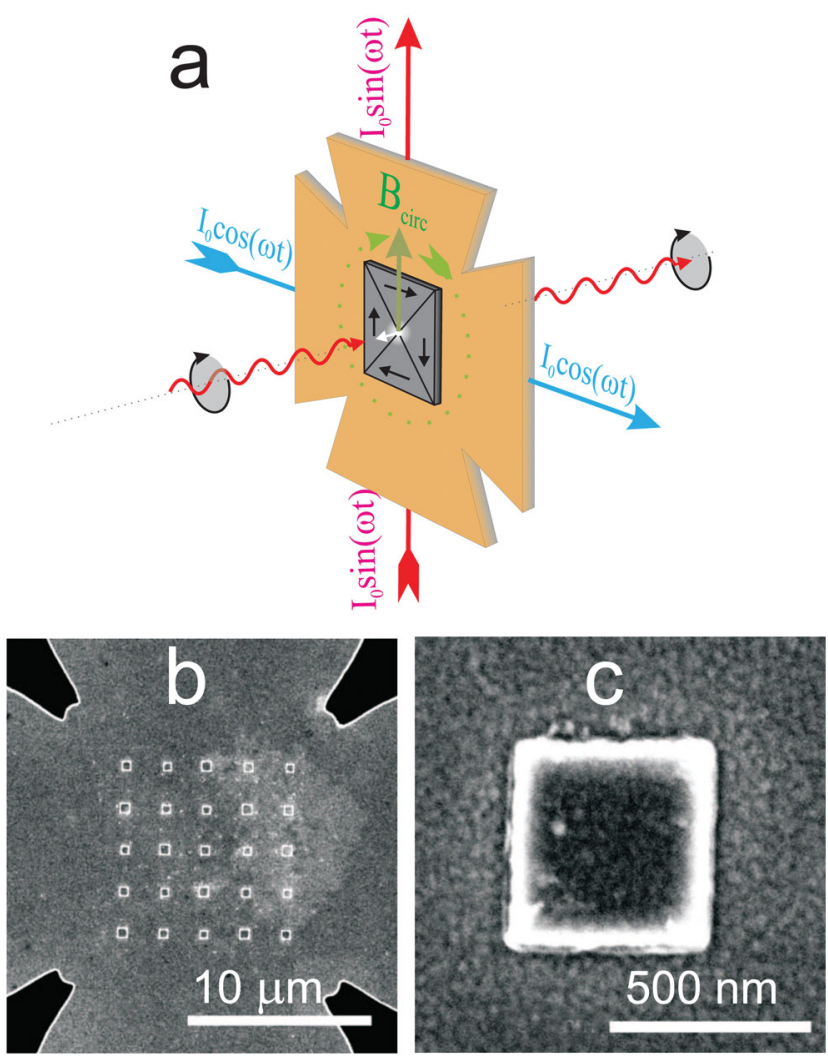

FIG. 1 (color). Schematic overview of the sample geometry. (a) The white arrow reflects the magnetic contrast of the "up" vortex core as given by the XMCD effect. Panel (b) shows a scanning electron micrograph (SEM) of the crossed strip line with an array of Py elements. Panel (c) shows a SEM micrograph of the single $500 \mathrm{~nm} \times 500 \mathrm{~nm} \times 50 \mathrm{~nm}$ Py element at the center of the array of which the x-ray absorption image are shown in Fig. 2.

currents with a $90^{\circ}$ phase difference to both arms of the crossed striplines. Each stripline was driven through a balun to ensure equal currents in both ends of the stripline in order to isolate the signal from the other arm. By changing the phase of one of the two signals by $180^{\circ}$, the rotation sense of the field can easily be changed from counterclockwise (CCW, $\omega>0)$ to clockwise $(\mathrm{CW}, \omega<$ $0)$. Because the generated magnetic field is not completely homogeneous over the stripline, the rotating field will effectively be elliptical away from the center of the cross. Based on finite element calculations of the current and magnetic field distributions, we have estimated this difference between the major and minor axes of the ellipse to be less then $3 \%$ for the investigated elements.

The time-dependent magnetization distribution of the sample was imaged by a stroboscopic measurement technique, with a scanning transmission $\mathrm{x}$-ray microscope at the Advanced Light Source (ALS, beam line 11.0.2) [11]. The contrast mechanism used for the imaging of the magnetic structure is the $\mathrm{x}$-ray magnetic circular dichroism (XMCD) effect, namely, the dependence of the absorption coefficient of circularly polarized monochromatic x-rays on the direction of the magnetization in a ferromagnetic sample [12]. The elliptically polarized monochromatic $\mathrm{x}$ rays provided by the undulator beam line are focused by a Fresnel zone plate to a spot of about $30 \mathrm{~nm}$. The sample is scanned through the focal spot with a highresolution scanning stage while the transmitted photon flux is measured. The data were recorded at the $\mathrm{Ni}$ $L 3$-absorption edge $(852.7 \mathrm{eV})$, at which the XMCD effect gives a strong magnetic contrast. For right circularly polarized light, the absorbed $\mathrm{x}$-ray intensity is proportional to the projection of the magnetization vector $\mathbf{M}$ on the photon propagation vector $\mathbf{k}$. When the sample is placed perpendicular to the photon beam (see Fig. 1), only the out-ofplane magnetization contributes to the magnetic contrast, so the vortex core can be directly imaged and its polarization can be derived. Alternatively, the sample normal was tilted by $30^{\circ}$ with respect to the photon beam to image the in-plane magnetization of the investigated Landau structures. In this geometry, the observed magnetic contrast is dominated by the contribution from the $x$ component of the in-plane closed flux magnetization and the circulation of the vortex structure could be verified.

To record snapshots of the magnetic response in a stroboscopic fashion, the oscillating excitation field is synchronized with the probing $\mathrm{x}$-ray flashes. This allows us to image the magnetization with a time resolution of $70 \mathrm{ps}$, as given by the width of the x-ray flashes. An excitation frequency of $562.5 \mathrm{MHz}$ was chosen, which is $9 / 8$ of the photon flash repetition frequency and close to the gyrotropic resonance frequency. In this way, we can simultaneously record the response at 8 different phases of the excitation by using an 8-channel acquisition [13].

Figure 2 shows the result of the sequence of magnetic fields that was applied to one of the vortex structures. The corresponding movies are available online [14]. A clockwise $(\mathrm{CW})$ rotating field was applied to a vortex down state (Fig. 2, row a, $p=-1$ ). This rotation sense coincides with the gyrotropic rotation sense of the core and the core gyration can clearly be observed. When the amplitude of the rotating field is increased, the gyration amplitude increases (row b), until at an amplitude of $0.385 \mathrm{mT}$ the polarization of the vortex core reverses and the gyration stops (row c). Even when the amplitude was decreased again, the vortex appears static. Now, the same procedure was repeated with a counterclockwise $(\mathrm{CCW})$ rotating field to the vortex up state and a CCW gyration is observed (rows d, e) until at an rf field amplitude of $0.430 \mathrm{mT}$ the vortex motion stops again (row $\mathrm{f}$ ). This sequence was repeated many times and the response of the vortex always followed the same scheme.

These observations indicate that the core can be unidirectionally switched using rotating fields: an up vortex and a down vortex can be reversed by, respectively, a CCW and a $\mathrm{CW}$ rotating field of sufficient amplitude. When the core 


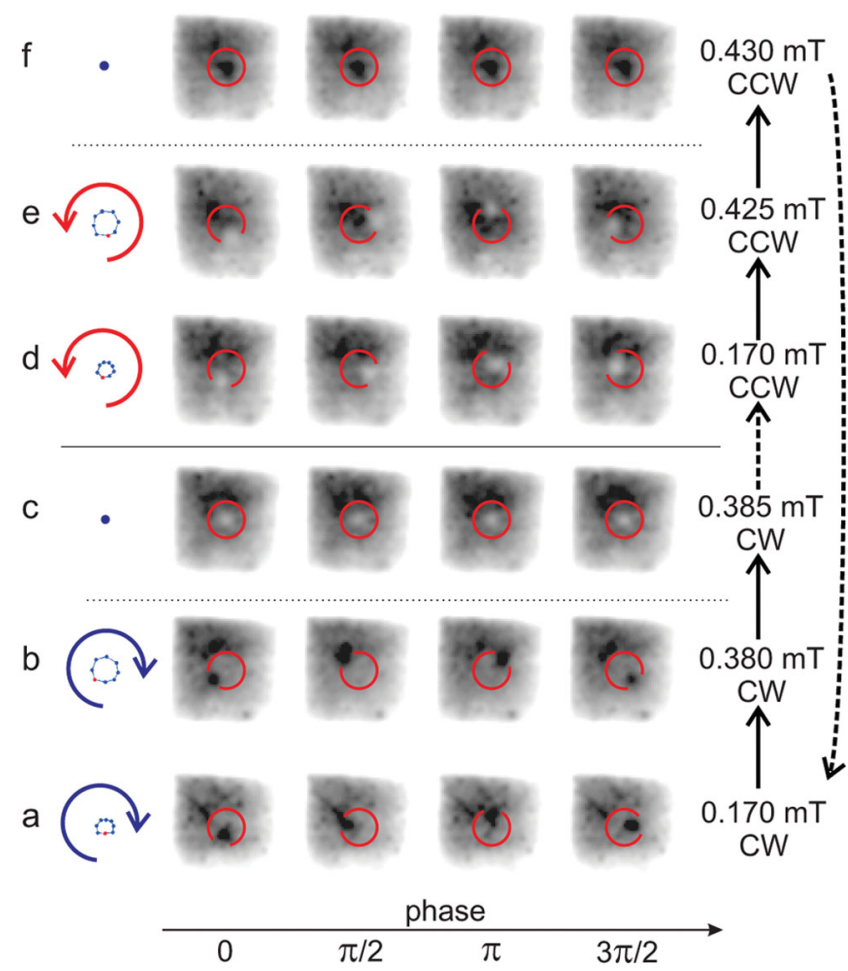

FIG. 2 (color). A sequence of time-resolved images of the vortex core with right circularly polarized photons in a $500 \times$ $500 \times 50 \mathrm{~nm}^{3}$ magnetic vortex structure excited by an in-plane rotating magnetic field. The out-of-plane magnetization of the vortex core gives rise to a bright or dark spot, depending if the polarization of the core is positive or negative, respectively. The four frames in each row correspond to a full period of the applied counterclockwise (rows a-c) and clockwise (rows d-f) rotating magnetic field. The red circles guide the eye to the vortex gyration center. On the left side the corresponding vortex core trajectories are given, derived from all 8 acquired movie frames. The movies are available online [14].

polarization has switched, the rotating field can no longer excite the vortex to the threshold velocity required for switching $[15,16]$ unless the rotation sense of the field is reversed as well.

Our experimental results agree qualitatively with those of the recent micromagnetic simulations [10] and with our own micromagnetic simulations. The OOMMF code [17] was used to perform 2-dimensional micromagnetic simulations with Py material parameters [18]. A magnetic element with a vortex structure having the same size as in the experiments and a core polarization $p=1$ was excited by a CCW field rotating with a frequency of the gyrotropic resonance. When the field amplitude exceeded $0.55 \mathrm{mT}$, a core reversal was observed via the formation and annihilation of vortex-antivortex pairs as first identified by Waeyenberge et al. [6]. Figure 3 shows the vortex core trajectories extracted from this simulation with a $4 \mathrm{mT}$ rotating field. We observe that the vortex is quickly accelerated counterclockwise by the applied field until the core
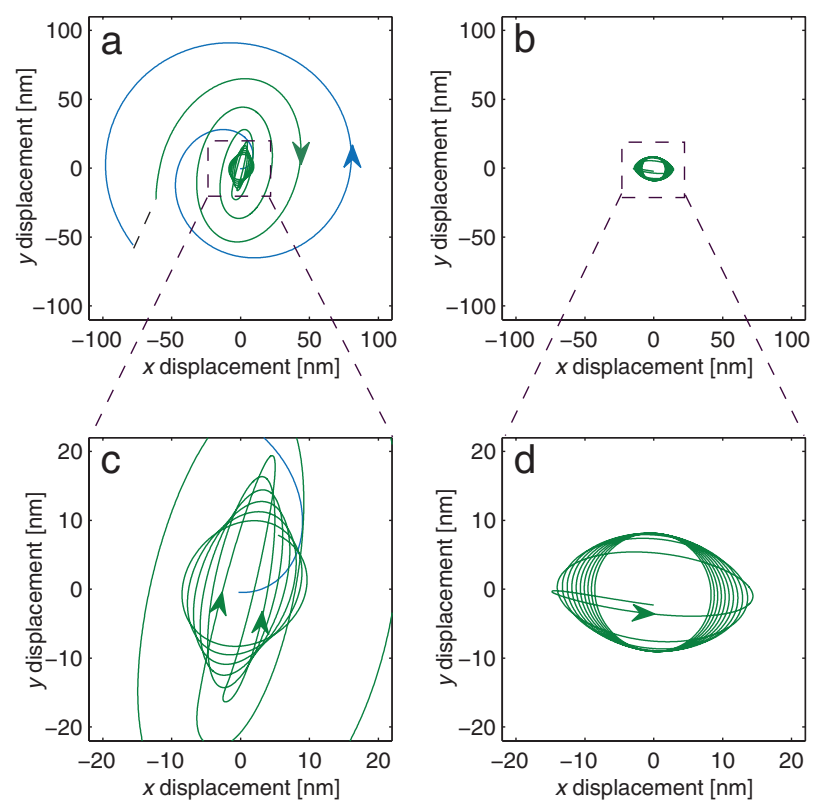

FIG. 3 (color). Trajectories obtained from micromagnetic simulations; panel (a) and (c): a vortex with initial core polarization $p=+1$ excited by a $4 \mathrm{mT} \mathrm{CCW}$ rotating field; panel (b) and (d): a vortex with core polarization $p=-1$ excited by a $4 \mathrm{mT}$ CCW rotating field. The colors indicate the vortex polarization, green $=$ down, blue $=$ up.

polarization switches. Now the vortex is decelerating, i.e., spiraling back clockwise toward the center of the structure. Here, the rotation sense changes sign again and only a small CCW motion remains. The same small motion without the switching event is observed when such a $4 \mathrm{mT}$ $\mathrm{CCW}$ rotating field is applied to a vortex with a down polarization, so opposite to the gyrotropic rotation sense. Only when the amplitude of a field is increased above $22 \mathrm{mT}$, the vortex core switches, but keeps switching back and forth. These simulations are consistent with the experimental observations if we consider that the small motion induced by a rotating field with the opposite sense of the vortex gyration is below the resolution of the microscope.

From the measured vortex core trajectories (see Fig. 2), the vortex velocity could be derived. At the low excitation amplitude of $0.17 \mathrm{mT}$, the velocity is about $120 \mathrm{~m} / \mathrm{s}$ for both vortex polarizations. At the higher excitation amplitudes, just below the switching threshold $(0.425 \mathrm{mT}$ for the up vortex and $0.38 \mathrm{mT}$ for the down vortex), the vortex velocity is about $190 \mathrm{~m} / \mathrm{s}$. This velocity is lower than the threshold velocity for switching of $320 \mathrm{~m} / \mathrm{s}$ estimated by Guslienko [16] and of $310 \mathrm{~m} / \mathrm{s}$ estimated from our own micromagnetic simulations. However, the experimental value is only a lower bound on the threshold velocity, as it is the average velocity over one gyration period. The peak velocity can be higher as roughness and/or the thermal fluctuations in the real sample might lead to fluctuations in the velocity. 
One observation was not reproduced by the simulations: the different switching thresholds for the two polarizations. In perfect samples, micromagnetic simulations show that the up and down vortex states are energetically equal and the dynamics are symmetric when, respectively, excited by a $\mathrm{CCW}$ and $\mathrm{CW}$ rotating magnetic field. A significant asymmetry between the two core polarizations has been observed before in a detailed measurement on the trajectories of the two core states [2]. Given the symmetry of the excitation field, such a $p$ asymmetry can only be understood if there exists an effective out-of-plane anisotropy. In the absence of out-of-plane magnetic fields, a possible cause could be a roughness induced shape anisotropy of which the opposite out-of-plane contributions do not cancel over the core trajectory. Another possible reason might be the Dzyaloshinskii-Moriya (DM) interaction [19,20] because of the lack of inversion symmetry in the Permalloy layer due to unlike top and bottom interfaces and/or to residual strain from the thin film deposition [21]. The lack of inversion symmetry gives rise to an interaction energy, $E_{\mathrm{DM}}=\sum_{i, j} \mathbf{D}_{i j} \cdot\left(\mathbf{S}_{i} \times \mathbf{S}_{j}\right)$, between the spin moments $\mathbf{S}$ at sites $i$ and $j$, where $\mathbf{D}_{i, j}$ is the DM vector and the cross product carries the information on the handedness of the vortex. Depending on the sign of $\mathbf{D}_{i j}$, the symmetry properties and vortex chirality, an up or down polarization may be preferred. However, it remains to be investigated, if the magnitude of these effects can cause the observed asymmetry.

To conclude, we showed that the fundamental eigenmodes of the two vortex core polarization states can be selectively excited by in-plane rotating magnetic fields. Such fields of sufficient strength can switch the vortex polarity in a unidirectional way. By changing the rotation sense of the field, vortices with either an up or down core magnetization can be excited. The experimental results show an asymmetry in the threshold amplitudes of the two polarization states for switching and suggest a breaking of symmetry.

Cooperation with Aleksandar Puzic, Kang Wei Chou, and Michael Hirscher is gratefully acknowledged. We would also like to thank Sabine Seiffert and Christian Wolter for the mechanical construction of the sample holder. A. V. acknowledges the financial support by The Institute for the Promotion of Innovation by Science and Technology in Flanders (IWT Flanders). The Advanced Light Source is supported by the Director, Office of Science, Office of Basic Energy Sciences, of the U.S. Department of Energy. *curcic@mf.mpg.de

+Bartel.VanWaeyenberge@UGent.be

"stoll@mf.mpg.de

[1] A. Wachowiak, J. Wiebe, M. Bode, O. Pietzsch, M. Morgenstern, and R. Wiesendanger, Science 298, 577 (2002).

[2] K. W. Chou, A. Puzic, H. Stoll, D. Dolgos, G. Schutz, B. Van Waeyenberge, A. Vansteenkiste, T. Tyliszczak, G. Woltersdorf, and C.H. Back, Appl. Phys. Lett. 90, 202505 (2007).

[3] A. A. Thiele, Phys. Rev. Lett. 30, 230 (1973).

[4] D. Huber, J. Appl. Phys. 53, 1899 (1982).

[5] B.E. Argyle, E. Terrenzio, and J. C. Slonczewski, Phys. Rev. Lett. 53, 190 (1984).

[6] B. Van Waeyenberge et al., Nature (London) 444, 461 (2006).

[7] K.-S. Lee and S.-K. Kim, Phys. Rev. B 78, 014405 (2008).

[8] J. Zagorodny, Y. Gaididei, F. Mertens, and A. Bishop, Eur. Phys. J. B 31, 471 (2003).

[9] V.P. Kravchuk, D.D. Sheka, Y. Gaididei, and F. G. Mertens, J. Appl. Phys. 102, 043908 (2007).

[10] S.-K. Kim, K.-S. Lee, Y.-S. Yu, and Y.-S. Choi, Appl. Phys. Lett. 92, 022509 (2008).

[11] A. L. D. Kilcoyne et al., J. Synchrotron Radiat. 10, 125 (2003).

[12] G. Schütz, W. Wagner, W. Wilhelm, P. Kienle, R. Zeller, R. Frahm, and G. Materlik, Phys. Rev. Lett. 58, 737 (1987).

[13] Y. Acremann, V. Chembrolu, J. P. Strachan, T. Tyliszczak, and J. Stöhr, Rev. Sci. Instrum. 78, 014702 (2007).

[14] See EPAPS Document No. E-PRLTAO-101-037844 for the movies of the data presented in Fig. 2. For more information on EPAPS, see http://www.aip.org/pubservs/ epaps.html.

[15] K. Yamada, S. Kasai, Y. Nakatani, K. Kobayashi, H. Kohno, A. Thiaville, and T. Ono, Nature Mater. 6, 269 (2007).

[16] K. Y. Guslienko, K.-S. Lee, and S.-K. Kim, Phys. Rev. Lett. 100, 027203 (2008).

[17] M. J. Donahue and D. G. Porter, National Institute of Standards and Technology Interagency Report No. NISTIR 6376, 1999.

[18] Simulation parameters: saturation magnetization $M_{s}=$ $760 \times 10^{3} \mathrm{~A} / \mathrm{m}$, exchange stiffness $A=13 \times 10^{-12} \mathrm{~J} / \mathrm{m}$, crystalline anisotropy constant $K_{1}=0$, damping parameter $\alpha=0.01$, cell size in $x$ and $y$ direction was $2 \mathrm{~nm}$, in the $z$ direction $50 \mathrm{~nm}$, excitation frequency $|\omega /(2 \pi)|=$ $562.5 \mathrm{MHz}$.

[19] I. E. Dzyaloshinskii, Sov. Phys. JETP 5, 1259 (1957).

[20] T. Moriya, Phys. Rev. 120, 91 (1960).

[21] A. N. Bogdanov and U.K. Rössler, Phys. Rev. Lett. 87, 037203 (2001). 